\title{
HIV Care and Viral Suppression During the Last Year of Life: A Comparison of HIV-Infected Persons Who Died of HIV-Attributable Causes With Persons Who Died of Other Causes in 2012 in 13 US Jurisdictions
}

William K Adih, DrPH, MPH, MD; H Irene Hall, PhD; Richard M Selik, MD; Xiuchan Guo, MD

Division of HIV/AIDS Prevention, National Center for HIV/AIDS, Viral Hepatitis, STD, and TB Prevention, Centers for Disease Control and Prevention, Atlanta, GA, United States

\section{Corresponding Author:}

William K Adih, DrPH, MPH, MD

Division of HIV/AIDS Prevention

National Center for HIV/AIDS, Viral Hepatitis, STD, and TB Prevention

Centers for Disease Control and Prevention

1600 Clifton Road, NE, Mailstop E-47

Atlanta, GA, 30333

United States

Phone: 14046395382

Fax: 14046392980

Email: wadih@cdc.gov

\section{Abstract}

Background: Little information is available about care before death among human immunodeficiency virus (HIV)-infected persons who die of HIV infection, compared with those who die of other causes.

Objective: The objective of our study was to compare HIV care and outcome before death among persons with HIV who died of HIV-attributable versus other causes.

Methods: We used National HIV Surveillance System data on CD4 T-lymphocyte counts and viral loads within 12 months before death in 2012, as well as on underlying cause of death. Deaths were classified as "HIV-attributable" if the reported underlying cause was HIV infection, an AIDS-defining disease, or immunodeficiency and as attributable to "other causes" if the cause was anything else. Persons were classified as "in continuous care" if they had $\geq 2$ CD4 or viral load test results $\geq 3$ months apart in those 12 months and as having "viral suppression" if their last viral load was <200 copies $/ \mathrm{mL}$.

Results: Among persons dying of HIV-attributable or other causes, respectively, 65.28\% (2104/3223) and 30.88\% (1041/3371) met AIDS criteria within 12 months before death, and 33.76\% (1088/3223) and 50.96\% (1718/3371) had viral suppression. The percentage of persons who received $\geq 2$ tests $\geq 3$ months apart did not differ by cause of death. Prevalence of viral suppression for persons who ever had AIDS was lower among those who died of HIV but did not differ by cause for those who never had AIDS.

Conclusions: The lower prevalence of viral suppression among persons who died of HIV than among those who died of other causes implies a need to improve viral suppression strategies to reduce mortality due to HIV infection.

(JMIR Public Health Surveill 2017;3(1):e3) doi: 10.2196/publichealth.6206

\section{KEYWORDS}

HIV; AIDS; causes of death; care

\section{Introduction}

As human immunodeficiency virus (HIV)-infected persons are surviving to older ages, the spectrum of causes of death among them is changing - the proportion of deaths in which HIV infection was the underlying cause has decreased, while the proportion of deaths due to causes not clearly attributable to HIV has increased [1-3]. Retention in medical care and effective treatment to achieve a suppressed viral load are essential to reduce morbidity and mortality, as well as the potential for onward transmission of the virus [4]. However, little information is available about care and outcomes before death among 
persons living with HIV who eventually died of either HIV-attributable or other causes. Assessment of predeath care by cause of death can inform opportunities for intervention. This study is an update on a recent study that examined HIV care within the year before death [5]. We expanded the investigation to compare HIV-infected persons who died of an HIV-attributable underlying cause with those who died of another underlying cause with respect to (1) disease stage within 12 months before death and (2) measures of care in terms of frequency of CD4 T-lymphocyte counts or viral load measurements and viral suppression.

\section{Methods}

All US states and the District of Columbia require reporting of cases of HIV infection to their health departments; however, not all have mandatory reporting of all values of CD4 T-lymphocyte cell counts and viral load test results by laboratories. We used data reported to the National HIV Surveillance System (NHSS) of the Centers for Disease Control and Prevention through July 2015 from 13 jurisdictions with mandated laboratory reporting of all results from HIV-related tests to their HIV surveillance programs and that also collected cause of death information (California, District of Columbia, Hawaii, Iowa, Louisiana, Maryland, Michigan, Missouri, New Hampshire, New York, South Carolina, Texas, West Virginia). The analysis was restricted to persons who died in 2012, were $\geq 13$ years old at the time of death, and resided in the selected areas at both diagnosis and death. The purpose of the residential restriction was to enhance the completeness of data on laboratory test results, because laboratories report test results to the health departments of the jurisdictions corresponding to the patient's residential address reported by the health care provider. Among the health departments of these 13 jurisdictions, at least $85 \%$ of the deaths they reported to NHSS had data on the underlying cause, which they obtained by linking HIV surveillance data with either state or local vital records data or the National Death Index. The health departments reported the data to NHSS without key personal identifiers (eg, name, Social Security number) that they used to link HIV cases to death records and laboratory test results. Causes of death were identified by codes in the International Classification of Diseases, Tenth Revision (ICD-10) [6]. We classified a death as "HIV-attributable" if the reported underlying cause was HIV infection, indicated either explicitly (by an ICD code for HIV infection itself) or implicitly (by an ICD code for an AIDS-defining opportunistic illness or immunodeficiency - cell-mediated or unspecified type, not an antibody-mediated or congenital type). We assumed HIV infection was underlying an opportunistic illness or immunodeficiency because all the decedents had HIV infection reported to NHSS, even if HIV was not mentioned on the death certificate. If the underlying cause was known but was not HIV infection, we classified the death as "non-HIV-attributable death" (and the underlying cause as "other" than HIV infection; Multimedia Appendix 1). Persons missing information on underlying causes of death were excluded from the analyses. Stage of disease was based on the most recent CD4 cell count or percentage, and was defined in a reversible way, so that a person whose HIV disease had previously met the criteria for stage 3 (AIDS) could be reclassified in stage 1 if the most recent CD 4 cell count was $\geq 500$ cells/ $\mu L$ [7]. Persons were considered to be "in care" within 12 months before death if they had $\geq 1$ CD4 or viral load test result in that period and "in continuous care" if they had $\geq 2 \mathrm{CD} 4$ or viral load test results at least 3 months apart within the last 12 months before death [8]. Viral suppression (defined as <200 copies/mL) was based on the most recent viral load in the 12 months before death.

We assessed indicators overall and by sex, age, race/ethnicity, and transmission category (male-to-male sexual contact, ie, men who had sex with men or MSM; injection drug use; MSM and injection drug use; heterosexual contact; and other). We also determined care and viral suppression by disease severity (whether a person's infection had ever been classified as stage 3 disease, AIDS), the length of time since diagnosis of HIV infection, and urban versus rural area of residence at the time of diagnosis (metropolitan statistical area population $\geq 500,000$; metropolitan statistical area population 50,000-499,999; and nonmetropolitan population <50,000). Using log-binomial regression, we calculated prevalence ratios and $95 \%$ CIs to determine statistical differences on measures of care between persons who died of HIV-attributable underlying causes and those who died of non-HIV-attributable underlying causes. To control for potential confounding covariates, the analyses were adjusted for sex, age at death, race/ethnicity, transmission category, and ever AIDS. Analyses were adjusted for missing risk factor information. Analyses were performed using SAS version 9.3 statistical software (SAS Institute Inc), with the GenMod procedure for the log-binomial regression.

\section{Results}

Among 6594 persons who died in 2012 with diagnosed HIV infection, $48.88 \%(3223 / 6594)$ died of HIV-attributable underlying causes and $51.12 \% \quad(3371 / 6594)$ died of non-HIV-attributable underlying causes. Of those who died of HIV-attributable causes, most were male (2374/3223, 73.66\%) and $\geq 40$ years old at death $(2673 / 3223,82.94 \%)$; their racial/ethnic distribution was $47.35 \%(1526 / 3223)$ non-Hispanic black or African American, 23.30\% (751/3223) non-Hispanic white, $22.25 \%(717 / 3223)$ Hispanic or Latino, and $7.11 \%$ (229/3223) other; 40.80\% (1315/3223) were MSM (Table 1). Among those who died of non-HIV-attributable causes, most were male $(2493 / 3371,73.95 \%)$ and $\geq 40$ years old at death (3064/3371, 90.89\%); their racial/ethnic distribution was $45.57 \%$ (1536/3371) non-Hispanic black or African American, 29.01\% (978/3371) non-Hispanic white, 18.30\% (617/3371) Hispanic or Latino, and $7.12 \%$ (240/3371) other; $36.78 \%$ (1240/3371) were MSM (Table 2). Overall, the percentage of persons with late-stage disease (stage 3, AIDS, based on the most recent indicator, ie, CD4 test or opportunistic illness diagnosis) in the 12 months before death was more than twice as great among persons who died of $\mathrm{HIV}$-attributable causes $(65.28 \%$, 2104/3223; Table 1) as among persons who died of non-HIV-attributable causes (30.88\%, 1041/3371; Table 2). The percentage with stage 3 disease was similarly higher in almost all demographic groups and transmission categories of persons who died of HIV-attributable causes than in their counterparts who died of other causes. 
Table 1. Most recent stage of disease within 12 months before death, among persons aged $\geq 13$ years who died of HIV-attributable causes in 2012 , in 13 US jurisdictions.

\begin{tabular}{|c|c|c|c|c|c|c|c|c|c|c|}
\hline \multirow[t]{3}{*}{ Decedent characteristics } & \multicolumn{10}{|c|}{ Most recent stage ${ }^{\mathrm{a}}$ of disease before HIV-attributable death ${ }^{\mathrm{b}}$} \\
\hline & \multicolumn{2}{|l|}{ Total } & \multicolumn{2}{|c|}{$\begin{array}{l}\text { Stage } 1 \\
\left(\mathrm{CD} 4^{\mathrm{c}} \geq 500\right. \\
\text { cells/ } \mu \mathrm{L} \text { or } \geq 29 \%)\end{array}$} & \multicolumn{2}{|c|}{$\begin{array}{l}\text { Stage } 2(\mathrm{CD} 4200- \\
499 \text { cells/ } \mu \mathrm{L} \text { or } \\
14 \%-28 \%)\end{array}$} & \multicolumn{2}{|c|}{$\begin{array}{l}\text { Stage } 3 \text { (AIDS; } \\
\text { OI }^{\mathrm{d}} \text { or } \mathrm{CD} 4<200 \\
\text { cells } / \mu \mathrm{L} \text { or }<14 \% \text { ) }\end{array}$} & \multicolumn{2}{|c|}{ Unknown } \\
\hline & $\mathrm{n}$ & $\%^{\mathrm{e}}$ & $\mathrm{n}$ & $\%^{\mathrm{f}}$ & $\mathrm{n}$ & $\%^{\mathrm{f}}$ & $\mathrm{n}$ & $\%^{\mathrm{f}}$ & $\mathrm{n}$ & $\%^{\mathrm{f}}$ \\
\hline Total & 3223 & 100 & 244 & 7.6 & 542 & 16.8 & 2104 & 65.3 & 333 & 10.3 \\
\hline \multicolumn{11}{|l|}{ Sex } \\
\hline Male & 2374 & 73.7 & 175 & 7.4 & 394 & 16.6 & 1543 & 65.0 & 262 & 11.0 \\
\hline Female & 849 & 26.3 & 69 & 8.1 & 148 & 17.4 & 561 & 66.1 & 71 & 8.4 \\
\hline \multicolumn{11}{|l|}{ Age (years) at death, year-end 2012} \\
\hline $13-29$ & 154 & 4.8 & 7 & 4.5 & 18 & 11.7 & 121 & 78.6 & 8 & 5.2 \\
\hline $30-39$ & 396 & 12.3 & 8 & 2.0 & 40 & 10.1 & 321 & 81.1 & 27 & 6.8 \\
\hline $40-49$ & 914 & 28.4 & 58 & 6.3 & 112 & 12.3 & 666 & 72.9 & 78 & 8.5 \\
\hline $50-59$ & 1107 & 34.3 & 90 & 8.1 & 201 & 18.2 & 681 & 61.5 & 135 & 12.2 \\
\hline$\geq 60$ & 652 & 20.2 & 81 & 12.4 & 171 & 26.2 & 315 & 48.3 & 85 & 13.0 \\
\hline \multicolumn{11}{|l|}{ Race or ethnicity } \\
\hline American Indian or Alaska Native & 2 & 0.1 & 0 & 0 & 0 & 0 & 2 & 100 & 0 & 0 \\
\hline Asian & 20 & 0.6 & 0 & 0 & 1 & 5.0 & 17 & 85.0 & 2 & 10.0 \\
\hline Black or African American & 1526 & 47.3 & 112 & 7.3 & 239 & 15.7 & 1030 & 67.5 & 145 & 9.5 \\
\hline Hispanic or Latino ${ }^{\mathrm{g}}$ & 717 & 22.2 & 43 & 6 & 124 & 17.3 & 483 & 67.4 & 67 & 9.3 \\
\hline Native Hawaiian or other Pacific Islander & 3 & 0.1 & 0 & 0 & 0 & 0 & 2 & 66.7 & 1 & 33.3 \\
\hline White & 751 & 23.3 & 74 & 9.9 & 143 & 19.0 & 432 & 57.5 & 102 & 13.6 \\
\hline Multiple races & 204 & 6.3 & 15 & 7.4 & 35 & 17.2 & 138 & 67.6 & 16 & 7.8 \\
\hline \multicolumn{11}{|l|}{ Transmission category $^{\mathbf{h}}$} \\
\hline Male-to-male sexual contact $\left(\mathrm{MSM}^{\mathrm{i}}\right)$ & 1315 & 40.8 & 98 & 7.4 & 216 & 16.4 & 854 & 65.0 & 148 & 11.2 \\
\hline Male injection drug use & 513 & 15.9 & 43 & 8.3 & 86 & 16.8 & 333 & 64.7 & 52.7 & 10.3 \\
\hline Female injection drug use & 305 & 9.5 & 26 & 8.5 & 63 & 20.5 & 189 & 61.8 & 28 & 9.2 \\
\hline $\mathrm{MSM}^{\mathrm{i}}$ and injection drug use & 251 & 7.8 & 19 & 7.6 & 48 & 19.2 & 154 & 61.4 & 30 & 11.8 \\
\hline Male heterosexual contact & 271 & 8.4 & 12 & 4.4 & 42 & 15.4 & 188 & 69.3 & 30 & 10.9 \\
\hline Female heterosexual contact & 521 & 16.2 & 42 & 8.0 & 81 & 15.4 & 359 & 68.9 & 40 & 7.6 \\
\hline Other & 47 & 1.4 & 6 & 11.8 & 7 & 15.3 & 28 & 60.9 & 6 & 12.0 \\
\hline \multicolumn{11}{|l|}{ Ever AIDS } \\
\hline Yes & 2946 & 91.4 & 171 & 5.8 & 448 & 15.2 & 2104 & 71.4 & 223 & 7.6 \\
\hline No & 277 & 8.6 & 73 & 26.4 & 94 & 33.9 & 0 & 0 & 110 & 39.7 \\
\hline
\end{tabular}

${ }^{\text {a }}$ Stage of disease within 12 months before death based on most recent CD4 test performed.

${ }^{b}$ HIV-attributable deaths were those for which HIV infection, AIDS-indicative opportunistic illness, or immunodeficiency was the underlying cause.

${ }^{\mathrm{c}} \mathrm{CD} 4$ : $\mathrm{CD} 4{ }^{+} \mathrm{T}-$ lymphocyte count (cells/ $\left.\mu \mathrm{L}\right)$ or percentage.

${ }^{\mathrm{d}}$ OI: opportunistic illness (ie, AIDS-defining condition).

${ }^{\mathrm{e}}$ Column percent.

${ }^{\mathrm{f}}$ Row percent.

${ }^{\mathrm{g}}$ Hispanic or Latino can be of any race.

${ }^{\mathrm{h}}$ Data on transmission category statistically adjusted to account for missing transmission category.

i MSM: men who had sex with men. 
Table 2. Most recent stage of disease within 12 months before death, among persons aged $\geq 13$ years who died of non-HIV-attributable causes in 2012.

\begin{tabular}{|c|c|c|c|c|c|c|c|c|c|c|}
\hline \multirow[t]{3}{*}{ Decedent characteristics } & \multicolumn{10}{|c|}{ Most recent stage $\mathrm{e}^{\mathrm{a}}$ of disease before non-HIV-attributable death ${ }^{\mathrm{b}}$} \\
\hline & \multicolumn{2}{|l|}{ Total } & \multicolumn{2}{|c|}{$\begin{array}{l}\text { Stage } 1 \\
\left(\mathrm{CD} 4^{\mathrm{c}} \geq 500\right. \\
\text { cells } / \mu \mathrm{L} \text { or } \geq 29 \%)\end{array}$} & \multicolumn{2}{|c|}{$\begin{array}{l}\text { Stage } 2(\mathrm{CD} 4200- \\
499 \text { cells } / \mu \mathrm{L} \text { or } \\
14 \%-28 \%)\end{array}$} & \multicolumn{2}{|c|}{$\begin{array}{l}\text { Stage } 3 \text { (AIDS; } \\
\text { OI }^{\mathrm{d}} \text { or } \mathrm{CD} 4<200 \\
\text { cells } / \mu \mathrm{L} \text { or }<14 \% \text { ) }\end{array}$} & \multicolumn{2}{|c|}{ Unknown } \\
\hline & $\mathrm{n}$ & $\%^{\mathrm{e}}$ & $\mathrm{n}$ & $\%^{\mathrm{f}}$ & $\mathrm{n}$ & $\%^{\mathrm{f}}$ & $\mathrm{n}$ & $\%^{\mathrm{f}}$ & $\mathrm{n}$ & $\%^{\mathrm{f}}$ \\
\hline Total & 3371 & 100 & 686 & 20.4 & 987 & 29.3 & 1041 & 30.9 & 657 & 19.5 \\
\hline \multicolumn{11}{|l|}{ Sex } \\
\hline Male & 2493 & 74.0 & 482 & 19.3 & 741 & 29.7 & 782 & 31.4 & 488 & 19.6 \\
\hline Female & 878 & 26.0 & 204 & 23.2 & 246 & 28.0 & 259 & 29.5 & 169 & 19.2 \\
\hline \multicolumn{11}{|l|}{ Age (years) at death, year-end 2012} \\
\hline $13-29$ & 74 & 2.2 & 16 & 21.6 & 17 & 23.0 & 20 & 27.0 & 21 & 28.4 \\
\hline $30-39$ & 233 & 6.9 & 55 & 23.6 & 55 & 23.6 & 77 & 33.0 & 46 & 19.7 \\
\hline $40-49$ & 786 & 23.3 & 163 & 20.7 & 208 & 26.5 & 264 & 33.6 & 151 & 19.2 \\
\hline $50-59$ & 1292 & 38.3 & 259 & 20 & 393 & 30.4 & 416 & 32.2 & 224 & 17.3 \\
\hline$\geq 60$ & 986 & 29.2 & 193 & 19.6 & 314 & 31.8 & 264 & 26.8 & 215 & 21.8 \\
\hline \multicolumn{11}{|l|}{ Race or ethnicity } \\
\hline American Indian or Alaska Native & 2 & 0.1 & 0 & 0 & 0 & 0 & 1 & 50.0 & 1 & 50.0 \\
\hline Asian & 17 & 0.5 & 3 & 17.6 & 7 & 41.2 & 5 & 29.4 & 2 & 11.8 \\
\hline Black or African American & 1536 & 45.6 & 294 & 19.1 & 452 & 29.4 & 481 & 31.3 & 309 & 20.1 \\
\hline Hispanic or Latino ${ }^{\mathrm{g}}$ & 617 & 18.3 & 105 & 17.0 & 196 & 31.8 & 218 & 35.3 & 98 & 15.9 \\
\hline Native Hawaiian or other Pacific Islander & 3 & 0.1 & 1 & 33.3 & 0 & 0 & 2 & 66.7 & 0 & 0 \\
\hline White & 978 & 29 & 230 & 23.5 & 262 & 26.8 & 264 & 27.0 & 222 & 22.7 \\
\hline Multiple races & 218 & 6.5 & 53 & 24.3 & 70 & 32.1 & 70 & 32.1 & 25 & 11.5 \\
\hline \multicolumn{11}{|l|}{ Transmission category $^{h}$} \\
\hline Male-to-male sexual contact $\left(\mathrm{MSM}^{\mathrm{i}}\right)$ & 1240 & 36.8 & 263 & 21.2 & 371 & 29.9 & 373 & 30.1 & 234 & 18.8 \\
\hline Male injection drug use & 677 & 20.0 & 110 & 16.3 & 202 & 29.9 & 226 & 33.5 & 138 & 20.4 \\
\hline Female injection drug use & 397 & 11.8 & 85 & 21.3 & 121 & 30.5 & 112 & 28.2 & 79 & 20.0 \\
\hline $\mathrm{MSM}^{\mathrm{i}}$ and injection drug use & 287 & 8.5 & 56 & 19.6 & 85 & 29.5 & 94 & 32.8 & 52 & 18.1 \\
\hline Male heterosexual contact & 273 & 8.1 & 52 & 19.1 & 76 & 27.8 & 85 & 31.1 & 60 & 22.0 \\
\hline Female heterosexual contact & 471 & 14.0 & 118 & 24.9 & 122 & 26.0 & 143 & 30.4 & 88 & 18.8 \\
\hline Other & 27 & 0.8 & 3 & 11.7 & 11 & 39.6 & 7 & 26.7 & 6 & 22 \\
\hline \multicolumn{11}{|l|}{ Ever AIDS } \\
\hline Yes & 2564 & 76.1 & 401 & 15.6 & 779 & 30.4 & 1041 & 40.6 & 343 & 13.4 \\
\hline No & 807 & 23.9 & 285 & 35.3 & 208 & 25.8 & 0 & 0 & 314 & 38.9 \\
\hline
\end{tabular}

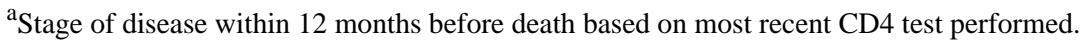

${ }^{b}$ Non-HIV-attributable deaths were all deaths for which the underlying cause was known other than those for which the underlying cause was HIV disease, an AIDS-indicative opportunistic illness, or immunodeficiency.

${ }^{\mathrm{c}} \mathrm{CD} 4$ : CD4 ${ }^{+} \mathrm{T}-$ lymphocyte count $($ cells/ $\mu \mathrm{L})$ or percentage.

${ }^{\mathrm{d}} \mathrm{OI}$ : opportunistic illness (ie, AIDS-defining condition).

${ }^{\mathrm{e}}$ Column percent.

${ }^{f}$ Row percent.

$\mathrm{g}_{\text {Hispanic or Latino can be of any race. }}$

${ }^{\mathrm{h}}$ Data on transmission category statistically adjusted to account for missing transmission category.

${ }^{\mathrm{i}}$ MSM: men who had sex with men. 
Overall, 91.34\% (2944/3223) and 82.74\% (2789/3371) of persons who died of HIV-attributable or non-HIV-attributable causes, respectively, had care within 12 months before death ( $\geq 1$ CD4 or viral load test; Table 3 ). The percentage of persons who received continuous care $(\geq 2$ CD4 or viral load tests, 3 months apart) was similar among persons who died of HIV-attributable and non-HIV-attributable causes (66.15\%, $2132 / 3223$ and $65.89 \%, 2221 / 3371$, respectively; Table 4). The percentage of persons with viral suppression was substantially lower among those who died of HIV-attributable causes (33.76\%, 1088/3223) than among those who died of non-HIV-attributable causes $(50.96 \%, 1718 / 3371$; adjusted prevalence ratio $0.69,95 \%$ CI $0.65-0.73$; Table 4). Prevalence of viral suppression for persons who ever had stage 3 (AIDS) was lower among those who died of HIV but did not differ by cause of death for persons who never had stage 3 (AIDS).
The percentages with $\geq 1$ CD4 or viral load test was $\geq 74 \%$ for most demographic groups and transmission categories but somewhat lower for persons who never had stage 3 disease (AIDS; 69.0\%, 191/277 and 64.7\%, 522/807 among persons who died of HIV-attributable and non-HIV-attributable causes, respectively) and persons whose diagnosis of HIV infection was $\leq 12$ months before their death due to non-HIV-attributable causes $(67.8 \%, 124 / 183)$. The percentage of persons with viral suppression was lower in most demographic and behavioral groups of persons who died of HIV-attributable causes than in their counterparts of persons who died of other causes.

Most persons in this study had HIV infection diagnosed more than 5 years before death $(2368 / 3223,73.47 \%$ and $2762 / 3371$, $81.93 \%$ among those who died of HIV-attributable and non-HIV-attributable causes, respectively; Table 3). 
Table 3. Care and viral suppression within 12 months before death, among persons aged $\geq 13$ years, comparing those who died of HIV-attributable causes with those who died of non-HIV-attributable causes in 2012, in 13 US jurisdictions: "in care" ( $\geq 1$ CD4 or viral load test).

\begin{tabular}{|c|c|c|c|c|c|c|c|c|c|c|c|c|}
\hline \multirow[t]{3}{*}{ Decedent characteristics } & \multirow{2}{*}{$\begin{array}{l}\text { Total } \\
\text { HIV } \\
\text { deaths }{ }^{b}\end{array}$} & \multirow{2}{*}{$\begin{array}{l}\text { Non- } \\
\text { HIV } \\
\text { deaths }^{c}\end{array}$} & \multicolumn{6}{|c|}{$\geq 1 \mathrm{CD} 4$ or $\mathrm{VL}^{\mathrm{a}}$ test } & \multicolumn{4}{|c|}{ Unknown if had CD4 or VL test } \\
\hline & & & \multicolumn{2}{|c|}{ HIV deaths } & \multicolumn{2}{|c|}{$\begin{array}{l}\text { Non-HIV } \\
\text { deaths }\end{array}$} & \multirow[t]{2}{*}{$\mathrm{APR}^{\mathrm{d}}$} & \multirow[t]{2}{*}{$95 \% \mathrm{CI}^{\mathrm{e}}$} & \multicolumn{2}{|c|}{ HIV deaths ${ }^{b}$} & \multicolumn{2}{|c|}{$\begin{array}{l}\text { Non-HIV } \\
\text { deaths }^{c}\end{array}$} \\
\hline & $\mathrm{n}$ & $\mathrm{n}$ & $\mathrm{n}$ & $\%$ & $\mathrm{n}$ & $\%$ & & & $\mathrm{n}$ & $\%$ & $\mathrm{n}$ & $\%$ \\
\hline Total & 3223 & 3371 & 2944 & 91.3 & 2789 & 82.7 & 1.05 & $1.03-1.06$ & 279 & 8.7 & 582 & 17.0 \\
\hline \multicolumn{13}{|l|}{ Sex } \\
\hline Male & 2374 & 2493 & 2159 & 90.9 & 2060 & 82.6 & 1.05 & $1.03-1.07$ & 215 & 9.1 & 433 & 17.4 \\
\hline Female & 849 & 878 & 785 & 92.5 & 729 & 83.0 & 1.05 & $1.02-1.09$ & 64 & 7.5 & 149 & 17.0 \\
\hline \multicolumn{13}{|l|}{ Age (years) at death, year-end 2012} \\
\hline $13-29$ & 154 & 74 & 146 & 94.8 & 55 & 74.3 & 1.02 & $0.92-1.14$ & 8 & 5.2 & 19 & 25.7 \\
\hline $30-39$ & 396 & 233 & 371 & 93.7 & 193 & 82.8 & 1.02 & $0.97-1.07$ & 25 & 6.3 & 40 & 17.2 \\
\hline $40-49$ & 914 & 786 & 848 & 92.8 & 655 & 83.3 & 1.06 & $1.03-1.10$ & 66 & 7.2 & 131 & 16.7 \\
\hline $50-59$ & 1107 & 1292 & 999 & 90.2 & 1095 & 84.8 & 1.03 & $1.00-1.06$ & 108 & 9.8 & 197 & 15.2 \\
\hline$\geq 60$ & 652 & 986 & 580 & 89.0 & 791 & 80.2 & 1.09 & $1.05-1.13$ & 72 & 11.0 & 195 & 19.8 \\
\hline \multicolumn{13}{|l|}{ Race or ethnicity } \\
\hline Black or African American & 1526 & 1536 & 1397 & 91.5 & 1254 & 81.6 & 1.05 & $1.02-1.08$ & 129 & 8.5 & 282 & 18.4 \\
\hline Hispanic or Latino ${ }^{\mathrm{f}}$ & 717 & 617 & 661 & 92.2 & 529 & 85.7 & 1.04 & $1.00-1.08$ & 56 & 7.8 & 88 & 14.3 \\
\hline White & 751 & 978 & 671 & 89.3 & 790 & 80.8 & 1.07 & $1.03-1.11$ & 80 & 10.7 & 188 & 19.2 \\
\hline Other races & 229 & 240 & 215 & 93.9 & 216 & 90.0 & 1.02 & $0.97-1.07$ & 14 & 6.1 & 24 & 10.0 \\
\hline \multicolumn{13}{|l|}{ Transmission category $^{\mathrm{g}}$} \\
\hline $\begin{array}{l}\text { Male-to-male sexual contact } \\
\left(\mathrm{MSM}^{\mathrm{h}}\right)\end{array}$ & 1315 & 1240 & 1198 & 91.1 & 1041 & 84.0 & 1.03 & $1.01-1.06$ & 117 & 8.9 & 199 & 16.0 \\
\hline Male injection drug use & 513 & 676 & 470 & 91.6 & 548 & 81.1 & 1.08 & $1.03-1.12$ & 43 & 8.4 & 128 & 18.9 \\
\hline Female injection drug use & 305 & 397 & 281 & 92.0 & 327 & 82.2 & 1.08 & $1.02-1.14$ & 24 & 7.9 & 70 & 17.6 \\
\hline $\mathrm{MSM}^{\mathrm{h}}$ and injection drug use & 251 & 287 & 225 & 89.6 & 240 & 83.7 & 1.05 & $0.98-1.12$ & 26 & 10.4 & 47 & 16.4 \\
\hline Male heterosexual contact & 271 & 273 & 246 & 90.6 & 215 & 79.1 & 1.06 & $1.00-1.12$ & 25 & 9.2 & 58 & 21.2 \\
\hline Female heterosexual contact & 521 & 471 & 486 & 93.2 & 394 & 83.6 & 1.04 & $0.99-1.08$ & 35 & 6.7 & 77 & 16.3 \\
\hline Other & 47 & 27 & 39 & 83.9 & 23 & 85.3 & 0.93 & $0.77-1.12$ & 8 & 17.0 & 4 & 14.8 \\
\hline \multicolumn{13}{|l|}{ Ever AIDS } \\
\hline Yes & 2946 & 2564 & 2753 & 93.4 & 2267 & 88.4 & 1.05 & $1.03-1.06$ & 193 & 6.6 & 297 & 11.6 \\
\hline No & 277 & 807 & 191 & 69.0 & 522 & 64.7 & 1.05 & $0.96-1.16$ & 86 & 31.0 & 285 & 35.3 \\
\hline \multicolumn{13}{|l|}{ Time since HIV diagnosis } \\
\hline$\leq 12$ months & 450 & 183 & 410 & 91.1 & 124 & 67.8 & 1.34 & $1.21-1.49$ & 40 & 8.9 & 59 & 32.2 \\
\hline 13-24 months & 108 & 93 & 100 & 92.6 & 77 & 82.8 & 1.12 & $1.00-1.24$ & 8 & 7.4 & 16 & 17.2 \\
\hline $3-5$ years & 297 & 333 & 281 & 94.6 & 251 & 75.4 & 1.26 & $1.17-1.34$ & 16 & 5.4 & 82 & 24.6 \\
\hline More than 5 years & 2368 & 2762 & 2153 & 90.9 & 2337 & 84.6 & 1.07 & $1.04-1.09$ & 215 & 9.1 & 425 & 15.4 \\
\hline \multicolumn{13}{|l|}{ MSA $^{i}$ at diagnosis } \\
\hline MSA (population $\geq 500,000$ ) & 2688 & 2937 & 2468 & 91.8 & 2445 & 83.2 & 1.05 & $1.03-1.07$ & 220 & 8.2 & 492 & 16.8 \\
\hline MSA (population 50,000-499,999) & 397 & 337 & 352 & 88.7 & 268 & 79.5 & 1.07 & $1.01-1.13$ & 45 & 11.3 & 69 & 20.5 \\
\hline $\begin{array}{l}\text { Nonmetropolitan area (population } \\
<50,000 \text { ) }\end{array}$ & 128 & 81 & 115 & 89.8 & 66 & 81.5 & 0.98 & $0.91-1.05$ & 13 & 10.2 & 15 & 18.5 \\
\hline
\end{tabular}




\begin{tabular}{|c|c|c|c|c|c|c|c|c|c|c|c|c|}
\hline \multirow[t]{3}{*}{ Decedent characteristics } & \multirow{2}{*}{$\begin{array}{l}\text { Total } \\
\text { HIV } \\
\text { deaths }\end{array}$} & \multirow{2}{*}{$\begin{array}{l}\text { Non- } \\
\text { HIV } \\
\text { deaths }^{c}\end{array}$} & \multicolumn{6}{|c|}{$\geq 1 \mathrm{CD} 4$ or $\mathrm{VL}^{\mathrm{a}}$ test } & \multicolumn{4}{|c|}{ Unknown if had CD4 or VL test } \\
\hline & & & \multicolumn{2}{|c|}{ HIV deaths } & \multicolumn{2}{|c|}{$\begin{array}{l}\text { Non-HIV } \\
\text { deaths }\end{array}$} & \multirow[t]{2}{*}{$\mathrm{APR}^{\mathrm{d}}$} & \multirow[t]{2}{*}{$95 \% \mathrm{Cl}^{\mathrm{e}}$} & \multicolumn{2}{|c|}{ HIV deaths ${ }^{b}$} & \multicolumn{2}{|c|}{$\begin{array}{l}\text { Non-HIV } \\
\text { deaths }^{c}\end{array}$} \\
\hline & $\mathrm{n}$ & $\mathrm{n}$ & $\mathrm{n}$ & $\%$ & $\mathrm{n}$ & $\%$ & & & $\mathrm{n}$ & $\%$ & $\mathrm{n}$ & $\%$ \\
\hline Unknown & 10 & 16 & 9 & 90.0 & 10 & 62.5 & - & & 1 & 10.0 & 6 & 37.5 \\
\hline
\end{tabular}

${ }^{\mathrm{a}}$ VL: viral load (copies/mL).

${ }^{\mathrm{b}} \mathrm{HIV}$ deaths (HIV-attributable deaths) were those for which HIV infection, AIDS-indicative opportunistic illness, or immunodeficiency was the underlying cause of death.

${ }^{\mathrm{c}}$ Non-HIV deaths (non-HIV-attributable deaths) were all other deaths for which the underlying cause was known.

${ }^{\mathrm{d}}$ APR: adjusted prevalence ratio, adjusted for sex, age at death, race/ethnicity, transmission category, and ever AIDS.

${ }^{\mathrm{e}} \mathrm{CI}$ : confidence interval

${ }^{\mathrm{f}}$ Hispanic or Latino can be of any race.

${ }^{\mathrm{g}}$ Data on transmission category have been statistically adjusted to account for missing transmission category.

hMSM: Men who had sex with men.

${ }^{\mathrm{i}}$ MSA: metropolitan statistical area. 
Table 4. Care and viral suppression within 12 months before death, among persons aged $\geq 13$ years, comparing those who died of HIV-attributable causes with those who died of non-HIV-attributable causes in 2012, in 13 US jurisdictions: "in continuous care" ( $\geq 2$ CD4 or viral load tests at least 3 months apart).

\begin{tabular}{|c|c|c|c|c|c|c|c|c|c|c|c|c|}
\hline \multirow[t]{3}{*}{ Decedent characteristics } & \multicolumn{6}{|c|}{$\geq 2 \mathrm{CD} 4$ or $\mathrm{VL}^{\mathrm{a}}$ test at least 3 months apart } & \multicolumn{6}{|c|}{ VL $<200$ copies/mL } \\
\hline & \multicolumn{2}{|c|}{ HIV deaths ${ }^{b, j}$} & \multicolumn{2}{|c|}{$\begin{array}{l}\text { Non-HIV } \\
\text { deaths } \\
\text { c,j }\end{array}$} & \multirow[t]{2}{*}{$\mathrm{APR}^{\mathrm{d}}$} & \multirow[t]{2}{*}{$95 \% \mathrm{CI}^{\mathrm{e}}$} & \multicolumn{2}{|c|}{ HIV deaths $s^{b, j}$} & \multicolumn{2}{|c|}{$\begin{array}{l}\text { Non-HIV } \\
\text { deaths }\end{array}$} & \multirow[t]{2}{*}{$\mathrm{APR}^{\mathrm{d}}$} & \multirow[t]{2}{*}{$95 \% \mathrm{CI}^{\mathrm{e}}$} \\
\hline & $\mathrm{n}$ & $\%$ & $\mathrm{n}$ & $\%$ & & & $\mathrm{n}$ & $\%$ & $\mathrm{n}$ & $\%$ & & \\
\hline Total & 2132 & 66.1 & 2221 & 65.9 & 0.96 & $0.92-0.99$ & 1088 & 33.8 & 1718 & 51.0 & 0.69 & $0.65-0.73$ \\
\hline \multicolumn{13}{|l|}{ Sex } \\
\hline Male & 1532 & 64.5 & 1642 & 65.9 & 0.93 & $0.89-0.97$ & 843 & 35.5 & 1324 & 53.1 & 0.69 & $0.64-0.74$ \\
\hline Female & 600 & 70.7 & 579 & 65.9 & 0.99 & $0.93-1.06$ & 245 & 28.9 & 394 & 44.9 & 0.69 & $0.61-0.79$ \\
\hline \multicolumn{13}{|l|}{ Age (years) at death, year-end 2012} \\
\hline $13-29$ & 97 & 63.0 & 41 & 55.4 & 0.94 & $0.72-1.23$ & 34 & 22.1 & 20 & 27.0 & 1.12 & $0.60-2.09$ \\
\hline $30-39$ & 248 & 62.6 & 129 & 55.4 & 0.99 & $0.86-1.15$ & 69 & 17.4 & 98 & 42.1 & 0.42 & $0.32-0.56$ \\
\hline $40-49$ & 607 & 66.4 & 497 & 63.2 & 0.97 & $0.90-1.04$ & 284 & 31.1 & 361 & 45.9 & 0.67 & $0.59-0.76$ \\
\hline $50-59$ & 750 & 67.8 & 888 & 68.7 & 0.95 & $0.90-1.00$ & 396 & 35.8 & 672 & 52.0 & 0.66 & $0.60-0.73$ \\
\hline$\geq 60$ & 430 & 66.0 & 666 & 67.5 & 0.95 & $0.88-1.01$ & 305 & 46.8 & 567 & 57.5 & 0.8 & $0.73-0.89$ \\
\hline \multicolumn{13}{|l|}{ Race or ethnicity } \\
\hline Black or African American & 1055 & 69.1 & 987 & 64.3 & 1.01 & $0.96-1.06$ & 453 & 29.7 & 678 & 44.1 & 0.71 & $0.64-0.78$ \\
\hline Hispanic or Latino ${ }^{f}$ & 464 & 64.7 & 459 & 74.4 & 0.89 & $0.83-0.96$ & 245 & 34.2 & 353 & 57.2 & 0.63 & $0.56-0.72$ \\
\hline White & 458 & 61.0 & 600 & 61.3 & 0.97 & $0.90-1.05$ & 309 & 41.1 & 559 & 57.2 & 0.72 & $0.65-0.80$ \\
\hline Other races & 155 & 67.7 & 175 & 72.9 & 0.9 & $0.80-1.00$ & 81 & 35.4 & 128 & 53.3 & 0.7 & $0.57-0.87$ \\
\hline \multicolumn{13}{|l|}{ Transmission category } \\
\hline $\begin{array}{l}\text { Male-to-male sexual contact } \\
\left(\mathrm{MSM}^{\mathrm{h}}\right)\end{array}$ & 813 & 61.8 & 806 & 65.0 & 0.9 & $0.85-0.96$ & 456 & 34.7 & 696 & 56.1 & 0.65 & $0.59-0.71$ \\
\hline Male injection drug use & 362 & 70.6 & 459 & 68.0 & 1.01 & $0.94-1.08$ & 202 & 39.4 & 349 & 51.6 & 0.78 & $0.69-0.89$ \\
\hline Female injection drug use & 230 & 75.3 & 268 & 67.5 & 1.05 & $0.96-1.16$ & 94 & 30.7 & 181 & 45.5 & 0.72 & $0.59-0.88$ \\
\hline $\mathrm{MSM}^{\mathrm{h}}$ and injection drug use & 171 & 68.2 & 199 & 69.3 & 0.95 & $0.84-1.06$ & 92 & 36.7 & 161 & 56.2 & 0.66 & $0.54-0.81$ \\
\hline Male heterosexual contact & 171 & 63.3 & 167 & 61.2 & 0.95 & $0.84-1.08$ & 82 & 30.3 & 109 & 40.0 & 0.79 & $0.62-1.00$ \\
\hline Female heterosexual contact & 354 & 68.0 & 303 & 64.4 & 0.95 & $0.87-1.04$ & 142 & 27.3 & 209 & 44.5 & 0.65 & $0.54-0.78$ \\
\hline Other & 30 & 65.2 & 18 & 65.6 & 0.86 & $0.63-1.18$ & 19 & 41.7 & 13 & 48.7 & 1.08 & $0.61-1.94$ \\
\hline \multicolumn{13}{|l|}{ Ever AIDS } \\
\hline Yes & 1986 & 67.4 & 1848 & 72.1 & 0.94 & $0.91-0.98$ & 970 & 32.9 & 1395 & 54.4 & 0.66 & $0.62-0.71$ \\
\hline No & 146 & 52.7 & 373 & 46.2 & 1.13 & $0.99-1.29$ & 118 & 42.6 & 323 & 40.0 & 1.05 & $0.90-1.23$ \\
\hline \multicolumn{13}{|l|}{ Time since HIV diagnosis } \\
\hline$\leq 12$ months & 116 & 25.8 & 48 & 26.2 & 0.97 & $0.73-1.30$ & 80 & 17.8 & 31 & 16.9 & 0.89 & $0.59-1.34$ \\
\hline 13-24 months & 75 & 69.4 & 66 & 71.0 & 0.99 & $0.80-1.22$ & 39 & 36.1 & 46 & 49.5 & 0.68 & $0.47-0.98$ \\
\hline $3-5$ years & 193 & 65.0 & 181 & 54.4 & 1 & $0.88-1.14$ & 103 & 34.7 & 139 & 41.7 & 0.9 & $0.72-1.12$ \\
\hline More than 5 years & 1748 & 73.8 & 1926 & 69.7 & 1.02 & $0.99-1.06$ & 866 & 36.6 & 1502 & 54.4 & 0.71 & $0.67-0.76$ \\
\hline \multicolumn{13}{|l|}{ MSA $^{i}$ at diagnosis } \\
\hline MSA (population $\geq 500,000$ ) & 1816 & 67.6 & 1977 & 67.3 & 0.96 & $0.93-1.00$ & 941 & 35.0 & 1520 & 51.8 & 0.7 & $0.66-0.75$ \\
\hline MSA (population 50,000-499,999) & 230 & 57.9 & 189 & 56.1 & 0.95 & $0.83-1.08$ & 108 & 27.2 & 144 & 42.7 & 0.69 & $0.56-0.86$ \\
\hline $\begin{array}{l}\text { Nonmetropolitan area (population } \\
<50,000)\end{array}$ & 79 & 61.7 & 47 & 58.0 & 0.9 & $0.72-1.11$ & 36 & 28.1 & 48 & 59.3 & 0.43 & $0.31-0.60$ \\
\hline
\end{tabular}




\begin{tabular}{|c|c|c|c|c|c|c|c|c|c|c|c|c|}
\hline \multirow[t]{3}{*}{ Decedent characteristics } & \multicolumn{6}{|c|}{$\geq 2 \mathrm{CD} 4$ or $\mathrm{VL}^{\mathrm{a}}$ test at least 3 months apart } & \multicolumn{6}{|c|}{ VL $<200$ copies/mL } \\
\hline & \multicolumn{2}{|c|}{ HIV deaths $s^{b, j}$} & \multicolumn{2}{|c|}{$\begin{array}{l}\text { Non-HIV } \\
\text { deaths }\end{array}$} & \multirow[t]{2}{*}{$\mathrm{APR}^{\mathrm{d}}$} & \multirow[t]{2}{*}{$95 \% \mathrm{CI}^{\mathrm{e}}$} & \multicolumn{2}{|c|}{ HIV deaths ${ }^{b, j}$} & \multicolumn{2}{|c|}{$\begin{array}{l}\text { Non-HIV } \\
\text { deaths }^{\mathrm{c}, \mathrm{j}}\end{array}$} & \multirow[t]{2}{*}{$\mathrm{APR}^{\mathrm{d}}$} & \multirow[t]{2}{*}{$95 \% \mathrm{CI}^{\mathrm{e}}$} \\
\hline & $\mathrm{n}$ & $\%$ & $\mathrm{n}$ & $\%$ & & & $\mathrm{n}$ & $\%$ & $\mathrm{n}$ & $\%$ & & \\
\hline Unknown & 7 & 70.0 & 8 & 50.0 & - & & 3 & 30.0 & 6 & 37.5 & - & \\
\hline
\end{tabular}

${ }^{\mathrm{a}} \mathrm{VL}$ : viral load (copies/mL).

${ }^{\mathrm{b}} \mathrm{HIV}$ deaths (HIV-attributable deaths) were those for which HIV infection, AIDS-indicative opportunistic illness, or immunodeficiency was the underlying cause of death.

${ }^{\mathrm{c}}$ Non-HIV deaths (non-HIV-attributable deaths) were all other deaths for which the underlying cause was known.

${ }^{\mathrm{d}}$ APR: adjusted prevalence ratio, adjusted for sex, age at death, race/ethnicity, transmission category, and ever AIDS.

${ }^{\mathrm{e}} \mathrm{CI}$ : confidence interval

${ }^{\mathrm{f}}$ Hispanic or Latino can be of any race.

g Data on transmission category have been statistically adjusted to account for missing transmission category.

hMSM: men who had sex with men.

${ }^{\mathrm{i}}$ MSA: metropolitan statistical area.

${ }^{\mathrm{j}}$ Total HIV and non-HIV deaths (denominators) are shown in Table 3.

\section{Discussion}

\section{Principal Findings}

Our results indicated poorer outcome in terms of viral suppression in the last 12 months before death among persons who died of HIV-attributable causes, consistent with other studies [9]. Almost two-thirds (65.28\%) of persons who died of HIV-attributable causes had late-stage disease (stage 3, AIDS) in the 12 months before death, compared with $30.88 \%$ of those who died of other causes, and the percentage with a suppressed viral load was lower among persons who died of HIV-attributable causes $(33.76 \%)$ than among those who died of other causes $(50.96 \%)$. This association between death due to HIV-attributable causes and both late-stage disease and lack of viral suppression could be explained by the latter two conditions being characteristic of late (delayed) diagnosis of HIV infection, inadequate care and treatment, inadequate adherence to medication regimens, or treatment failure $[9,10]$.

Although a high percentage of persons who died received care, a low percentage of them had viral suppression, particularly among persons who died of HIV-attributable causes. This is consistent with other studies $[9,10]$. Although receipt of care might be expected to lead to viral suppression, the causal relationship may actually be in the reverse direction — being "in care" or "in continuous care," as measured by frequency of CD4 or viral load tests, could be a marker for clinical deterioration with high viral loads, due to lack of adherence or treatment failure, which then resulted in more frequent care.

\section{Limitations}

Our analyses were subject to some limitations. First, our analyses were based on data from 13 jurisdictions, representing
$41 \%$ of all persons 13 years and older who died in 2012 in the United States, and, therefore, may not be representative of all persons with HIV who died in the United States. Second, cause of death information from death certificates may underestimate deaths due to HIV [11,12]. The finding that a substantial percentage $(30.88 \%)$ of the persons who died of non-HIV-attributable causes had stage 3 (AIDS) suggests that some of these deaths might actually have been HIV-attributable. Conversely, HIV infection may have been characterized as underlying cause of death for some patients without actually playing a role in their death if the physicians who certified the deaths ignored the instructions on the death certificate to list as causes only those conditions that "resulted in" or "contributed to" death, or incorrectly assumed that HIV infection did so. Third, information was not available on highly active antiretroviral therapy (HAART) or treatment adherence. Fourth, CD4 and viral load testing may not adequately capture the full spectrum of HIV care in the last 12 months before death [5].

\section{Conclusions}

HAART has prolonged the survival of HIV-infected persons by reducing deaths due to HIV-attributable causes [13]. To further decrease mortality, HIV-infected persons should seek early testing and, when diagnosed, be linked to care as soon as possible and be retained in such care so as to reduce the risk of death due to HIV and enable persons with HIV to have a life expectancy similar to that of persons without HIV [13]. This is in consonance with the recommendation by the Panel on Antiretroviral Guidelines for Adults and Adolescents that diagnosis of HIV be made early in the course of infection so as to initiate therapy early and at any CD4 count $[14,15]$.

\section{Acknowledgments}

The findings and conclusions in this article are those of the authors and do not necessarily represent the views of the Centers for Disease Control and Prevention. 


\title{
Conflicts of Interest
}

None declared.

\section{Multimedia Appendix 1}

[PDF File (Adobe PDF File), 43KB-Multimedia Appendix 1]

\section{References}

1. Adih WK, Selik RM, Hall HI, Babu AS, Song R. Associations and trends in cause-specific rates of death among persons reported with HIV infection, 23 US jurisdictions, through 2011. Open AIDS J 2016;10:144-157 [FREE Full text] [doi: 10.2174/1874613601610010144] [Medline: 27708746]

2. Adih WK, Selik RM, Hu X. Trends in diseases reported on US death certificates that mentioned HIV infection, $1996-2006$. J Int Assoc Physicians AIDS Care (Chic) 2011;10(1):5-11. [doi: 10.1177/1545109710384505] [Medline: 21088284]

3. Palella Jr FJ, Baker RK, Moorman AC, Chmiel JS, Wood KC, Brooks JT, HIV Outpatient Study Investigators. Mortality in the highly active antiretroviral therapy era: changing causes of death and disease in the HIV outpatient study. J Acquir Immune Defic Syndr 2006;43(1):27-34. [Medline: 16878047]

4. Centers for Disease Control and Prevention. Vital Signs: HIV prevention through care and treatment--United States. MMWR Mortal Wkly Rep 2011;60(47):1618-1623. [Medline: 22129997]

5. Hall HI, Espinoza L, Harris S, Shi J. Care and viral suppression during the last year of life among persons with HIV who died in 2012, 18 US jurisdictions. AIDS Care 2016 May;28(5):574-578. [doi: 10.1080/09540121.2015.1118428] [Medline: $\underline{26643945}$

6. World HO. ICD-10: International Classification of Diseases: Manual of the International Statistical Classification of Disease and Related Problems 10th Revision. Geneva: World Health Organization; 1992.

7. Centers for Disease Control and Prevention. Revised surveillance case definition for HIV infection--United States, 2014. MMWR Recomm Rep 2014;63(RR-03):1-10 [FREE Full text] [Medline: 24717910]

8. Whitehouse. National HIV/AIDS strategy for the United States. 2010. URL: https://www.whitehouse.gov/administration/ eop/onap/nhas [accessed 2017-01-11] [WebCite Cache ID 6nRTwtikE]

9. Braunstein S, Robbins R, Daskalakis D. Missed opportunities: Adapting the HIV care continuum to reduce HIV-related deaths. 2016 Feb 22 Presented at: Conference on Retroviruses and Opportunistic Infections. Abstract \#994; 2016; Boston, MA, USA URL: http://www1.nyc.gov/assets/doh/downloads/pdf/dires/2016-croi-missed-opportunities.pdf

10. Nassau T, Miller M, Branca S, Terrell C, Brady K. Receipt of Ryan White care services is associated with improved long-term outcomes. 2016 Feb 22 Presented at: Conference on RetrovirusesOpportunistic Infections. Abstract \#1002; 2016; Boston, MA, USA.

11. Chu SY, Buehler JW, Lieb L, Beckett G, Conti L, Costa S, et al. Causes of death among persons reported with AIDS. Am J Public Health 1993 Oct;83(10):1429-1432. [Medline: $\underline{8214233]}$

12. Hessol NA, Buchbinder SP, Colbert D, Scheer S, Underwood R, Barnhart JL, et al. Impact of HIV infection on mortality and accuracy of AIDS reporting on death certificates. Am J Public Health 1992 Apr;82(4):561-564. [Medline: 1546772]

13. Samji H, Cescon A, Hogg RS, Modur SP, Althoff KN, Buchacz K, North American AIDS Cohort Collaboration on Research and Design (NA-ACCORD) of IeDEA. Closing the gap: increases in life expectancy among treated HIV-positive individuals in the United States and Canada. PLoS One 2013 Dec;8(12):e81355 [FREE Full text] [doi: 10.1371/journal.pone.0081355] [Medline: 24367482]

14. Aidsinfo.nih. Guidelines for the use of antiretroviral agents in HIV-1-infected adults and adolescents. 2012. URL: https:/ /aidsinfo.nih.gov/contentfiles/lvguidelines/adultandadolescentgl.pdf [accessed 2017-01-11] [WebCite Cache ID 6nRVbMG7w]

15. Thompson MA, Aberg JA, Hoy JF, Telenti A, Benson C, Cahn P, et al. Antiretroviral treatment of adult HIV infection: 2012 recommendations of the International Antiviral Society-USA panel. J Am Med Assoc 2012 Jul 25;308(4):387-402. [doi: 10.1001/jama.2012.7961] [Medline: 22820792]

\author{
Abbreviations \\ HAART: highly active antiretroviral therapy \\ HIV: human immunodeficiency virus \\ ICD-10: International Classification of Diseases, Tenth Revision \\ MSM: men who had sex with men \\ NHSS: National HIV Surveillance System
}


Edited by P Sullivan; submitted 15.06.16; peer-reviewed by K Delaney, J McKenney, PG Farnham; comments to author 02.10.16; revised version received 21.10.16; accepted 27.11.16; published 24.01.17

Please cite as:

Adih WK, Hall HI, Selik RM, Guo X

HIV Care and Viral Suppression During the Last Year of Life: A Comparison of HIV-Infected Persons Who Died of HIV-Attributable Causes With Persons Who Died of Other Causes in 2012 in 13 US Jurisdictions

JMIR Public Health Surveill 2017;3(1):e3

URL: http://publichealth.jmir.org/2017/1/e3/

doi: 10.2196/publichealth.6206

PMID: 28119277

(C) William K Adih, H Irene Hall, Richard M Selik, Xiuchan Guo. Originally published in JMIR Public Health and Surveillance (http://publichealth.jmir.org), 24.01.2017. This is an open-access article distributed under the terms of the Creative Commons Attribution License (http://creativecommons.org/licenses/by/2.0/), which permits unrestricted use, distribution, and reproduction in any medium, provided the original work, first published in JMIR Public Health and Surveillance, is properly cited. The complete bibliographic information, a link to the original publication on http://publichealth.jmir.org, as well as this copyright and license information must be included. 